\title{
Revisión conceptual de la conexión entre inteligencia emocional y autoconcepto físico
}

\section{Conceptual review of the connection between emotional intelligence and physical activity}

\author{
Félix Zurita Ortega, Ruben Moreno Arrebola, Gabriel González Valero, Virginia Viciana Garófano, \\ Asuncion Martínez Martínez y Jose Joaquín Muros Molina*
}

Departamento de Didáctica de la Expresión Musical, Plástica y Corporal de la Universidad de Granada (España)

\begin{abstract}
Resumen: En la actualidad el conocimiento de los aspectos físicos y cognitivos es esencial en el contexto deportivo, ante esta situación la imagen que tiene una persona sobre sí mismo (autoconcepto) y el cómo se encuentra emocionalmente (inteligencia emocional) repercute en la acción deportiva. Ante esta situación se plantea el siguiente trabajo con la intención de examinar el número de estudios que hay en la Web of Science que contengan los términos autoconcepto e inteligencia emocional junto con los parámetros actividad física y deporte, tanto en castellano como en inglés. Los resultados depararon que existía un mayor número de artículos en lengua inglesa que en castellano, asimismo los estudios relacionados con autoconcepto son más numerosos que los de inteligencia emocional, y cuando se buscan trabajos que contengan la totalidad de los parámetros antes citados, en castellano no se detectan trabajos, mientras que en inglés aparecen dieciséis. Palabras Clave: Deporte; Autoconcepto; InteligenciaEmocional; Actividad Física.
\end{abstract}

Abstract: At present the knowledge of the physical and cognitive aspects is essential in the sport context, before this situation the image that a person has about himself (self-concept) and how he is emotionally (emotional intelligence) has repercussions on sports action. Given this situation, the following work is proposed with the intention of examining the number of studies in the Web of Science that contain the terms self-concept and emotional intelligence along with the parameters physical activity and sport, in both Spanish and English. The results showed that there was a greater number of articles in English than in Spanish, and studies related to selfconcept are more numerous than those of emotional intelligence, and when searching for works that contain all of the aforementioned parameters, in Spanish no Jobs are detected, while in English sixteen appear.

Keywords: Sport; Self-Concept; Emotional Intelligence; Physical Activity.

\section{Introducción}

En la sociedad actual el conocimiento de la personalidad y la imagen que se tiene sobre uno mismo está experimentando un fuerte auge, en cuanto a número de investigaciones se refiere, en los últimos ańos (Soriano, Navas \& Holgado, 2014; Fuentes, 2014; Sánchez-Alcaraz \& Mármol, 2014; Reigal, Becerra, Hernández \& Martín, 2014; Zurita, Castro, Álvaro, Rodríguez \& Pérez, 2016). El objeto de estudio conceptualiza una serie de términos o dimensiones (social, académico, emocional, familiar o físico), que justifican la imagen que una persona puede tener de sí mismo.

Concretamente el autoconcepto de tipo físico que según lo planteado por Strein (1996) es: "la percepción que tienen los sujetos sobre sus habilidades físicas y apariencia física”, se encuentra presente en la mayoría de los modelos teóricos desde que se admitiera la multidimensionalidad y jerarquía del autoconcepto propuesta en su modelo teórico por Shavelson, Hubner\&Stanton (1976).

De esta forma y siguiendo las nuevas tendencias de teorías

Dirección para correspondencia [Correspodence address]: José Joaquín Muros Molina. Facultad de Ciencias de la Educación. Campus de Cartuja, Granada (España).Email: felixzo@ugr.es y jjmuros@ugr.es psicológicas sobre el autoconcepto, la mayoría concuerdan en que este término no constituye más que una construcción propia del individuo, y viene generada de la interacción de la persona con el entorno, que complementa lo planteado por otros científicos (Fox, 1988; García \& Musitu, 2001; Fernández, Contreras, García \& González, 2011).

El autoconcepto físico según lo planteado por Revuelta \& Esnaola (2011) viene determinado por elementos perceptivos y cognitivos (rasgos físicos, tamaño y formas del cuerpo), afectivos y emocionales (nivel de satisfacción), evaluativos, sociales o aspectos relacionados con las condiciones corporales (salud, atractivo físico o apariencia), en este sentido se entiende como: "una representación mental que se elabora al integrar la experiencia corporal y los sentimientos y emociones que esta produce".

Cuando los investigadores utilizan de manera indistinta ambos términos, autoconcepto y autoestima, se refieren al cúmulo de características, imágenes y sentimientos que el sujeto acepta como parte de sí mismo (Goñi 2009; De Tejada, 2010). Por lo que es un tema que aún se sigue debatiendo y estudiando, aunque bien es cierto que dos dimensiones (habilidad física y apariencia física) se encuentra recogidas en casi 
todos los modelos y si a esto le unimos la importancia que tiene la inteligencia emocional como uno de los aspectos que se deben de considerar y tener en cuenta en las habilidades y capacidades de las personas pues se puede considerar un elemento importante para su estudio.

La inteligencia emocional según Goleman (1995), es la capacidad que tiene el ser humano de controlar y regular los sentimientos de uno mismo, de los demás y emplear la emoción y el sentir como elemento de guía de los pensamientos y las acciones; otros autores como Mayer \& Salovey (1997) o Bar-On (2006), la definen como la habilidad o capacidad de percibir, integrar, comprender y manejar las emociones que tienen que ver con la comprensión de uno mismo y de los demás, y hacer frente con más éxito a las exigencias ambientales o externas. Los diversos autores consultados señalan la importancia de adquirir un mejor conocimiento de las emociones, saber denominarlas, ser capaz de identificarlas en sí mismo y en los demás, y lo que es más importante la capacidad de desarrollar la automotivación y fomentar la actitud positiva ante la vida (Zeidner, Matthews\& Roberts, 2008; Pineda, 2012).

Entre los múltiples autores consultados (Goleman, 1995; Salovey\& Mayer, 1997; Laborde, Guilien\& Watson, 2017), proponen parámetros que caracterizan al término propuesto, de esta manera componentes como la conciencia de uno mismo, la autoregulación, que permite en la persona el poder controlar y reconducir los impulsos y estados de ánimo, o el aspecto motivacional que trata de generar en la persona una situación donde se busquen las metas y no fijarse en los obstáculos. Otro de los elementos que se pueden considerar clave es la empatía, que es la habilidad que una persona tiene para entender las emociones de los demás y para tratar a las personas de acuerdo con sus reacciones emocionales, y finalmente la opción de las habilidades sociales, que consiste en construir relaciones con los demás y encontrar un espacio común para todos.

En los últimos años se ha constatado un amplio auge de los estudios que relacionan la inteligencia emocional con la actividad física, unos desde el prisma de la educación física (Ferrer-Caja \&Weiss, 2000), otros desde el deporte de competición (Ros-Martínez, Moya-Faz \& Garcés de los Fayos, 2013), y otros desde la visión de la psicología deportiva (Bretón, Zurita \& Cepero, 2017).

Lo que si se ha detectado es la escasez de trabajos que relacionan la actividad física con la inteligencia emocional y el autoconcepto físico, por lo que este trabajo pretende dar un paso en el conocimiento teórico y en conocer la relación que tiene el conocimiento de uno mismo con la inteligencia. En este trabajo teórico se tiene como objetivo examinar el número de estudios que hay en la Web of Science que contengan los términos de autoconcepto e inteligencia emocional junto con los parámetros de actividad física y deporte, tanto en cas- tellano como en inglés, así como determinar en qué rango de años se produce mayor índice de publicaciones.

\section{Método}

Para poder establecer un avance en el estudio del autoconcepto, inteligencia emocional y actividad física, según lo planteado anteriormente es importante conocer los resultados hallados y obtenidos por otros investigadores en este ámbito de estudio. Para ello se realiza una revisión teórica de los estudios siguiendo unos criterios, sustentados en que las investigaciones analizadas tuvieran una muestra específica relacionada con la actividad física o el deporte, ya que es bastante complejo que estudios realizados con personas no deportistas se extrapolen al contexto físico-deportivo tal y como plantean Bretón, Zurita \& Cepero (2016).

El procedimiento para la realización de estudios de este tipo tiene su punto fuerte en la base de datos en donde se realice la búsqueda, para este trabajo se realizará en la Web of Science, una vez dentro de la base se realizará con vocablos tanto en castellano como en lengua inglesa.

Los parámetros que se analizarán son: autoconcepto, inteligencia emocional, deporte y actividad física (castellano) y self-concept, emotional intelligence, sport y physical activity, de una manera individual y mediante las diversas combinaciones. Para la realización de este trabajo se consultó la base de datos anteriormente citadas en el primer trimestre del año 2017.

Otra de las distinciones que se realizará será en base a los años de publicación, agrupándose tres rangos: el primero de ellos abarcará hasta el 1999 inclusive, un segundo rango entre el 2000 y el 2010 inclusive y finalmente desde el 2011 hasta el año 2017.

\section{Resultados}

En la siguiente tabla se observa los datos concernientes a la búsqueda en las diversas base de datos, de esta se desprenden que el número de artículos en castellano $(\mathrm{n}=14)$, es muy inferior al de estudios escritos en inglés $(\mathrm{n}=5.183)$. Del mismo modo y tal como se aprecia de los 5.197 estudios analizados, 4.806 son relacionados con el autoconcepto y la actividad físico deportivo (self concept and physical activity/sport) y 365 son los que contienen en sus palabras claves la inteligencia emocional y el deporte.

En cuanto a la combinación de toda la terminología solamente 16 estudios contienen los tres parámetros objeto de estudio. Al estudiar por rango de años, aquellos trabajos publicados antes del año 1999 son muy inferiores $(n=808)$, a los que van desde el 2000 al $2010(n=2.270)$ y los del 2010 al $2017(n=2.119)$. 
Tabla 1. Búsqueda de parámetros en Web of Science.

\begin{tabular}{|c|c|c|c|}
\hline Palabras Clave & Años & Artículos & Total \\
\hline \multirow{3}{*}{ Autoconcepto y Deporte } & Hasta 1999 & 0 & \multirow{3}{*}{2} \\
\hline & $2000-2010$ & 1 & \\
\hline & Desde 2011 & 1 & \\
\hline \multirow{3}{*}{$\begin{array}{l}\text { Autoconcepto y Actividad } \\
\text { Física }\end{array}$} & Hasta 1999 & 0 & \multirow{3}{*}{8} \\
\hline & $2000-2010$ & 1 & \\
\hline & Desde 2011 & 7 & \\
\hline \multirow{3}{*}{$\begin{array}{l}\text { Inteligencia Emocional y } \\
\text { Deporte }\end{array}$} & Hasta 1999 & 0 & \multirow{3}{*}{3} \\
\hline & $2000-2010$ & 0 & \\
\hline & Desde 2011 & 3 & \\
\hline \multirow{3}{*}{$\begin{array}{l}\text { Inteligencia Emocional y Acti- } \\
\text { vidad Física }\end{array}$} & Hasta 1999 & 0 & \multirow{3}{*}{1} \\
\hline & $2000-2010$ & 0 & \\
\hline & Desde 2011 & 1 & \\
\hline \multirow{3}{*}{$\begin{array}{l}\text { Autoconcepto, Inteligencia } \\
\text { Emocional y Actividad Física }\end{array}$} & Hasta 1999 & 0 & \multirow{3}{*}{0} \\
\hline & $2000-2010$ & 0 & \\
\hline & Desde 2011 & 0 & \\
\hline \multirow{3}{*}{ Self-Concept and Sport } & Hasta 1999 & 344 & \multirow{3}{*}{1861} \\
\hline & $2000-2010$ & 837 & \\
\hline & Desde 2011 & 680 & \\
\hline \multirow{3}{*}{$\begin{array}{l}\text { Self-Concept and Physical } \\
\text { Activity }\end{array}$} & Hasta 1999 & 448 & \multirow{3}{*}{2945} \\
\hline & $2000-2010$ & 1334 & \\
\hline & Desde 2011 & 1163 & \\
\hline \multirow{3}{*}{$\begin{array}{l}\text { Emotional Intelligence and } \\
\text { Sport }\end{array}$} & Hasta 1999 & 2 & \multirow{3}{*}{193} \\
\hline & $2000-2010$ & 50 & \\
\hline & Desde 2011 & 141 & \\
\hline \multirow{3}{*}{$\begin{array}{l}\text { Emotional Intelligence and } \\
\text { Physical Activity }\end{array}$} & Hasta 1999 & 13 & \multirow{3}{*}{168} \\
\hline & $2000-2010$ & 42 & \\
\hline & Desde 2011 & 113 & \\
\hline \multirow{3}{*}{$\begin{array}{l}\text { Self-Concept Emotional Inte- } \\
\text { lligence and Physical Activity }\end{array}$} & Hasta 1999 & 1 & \multirow{3}{*}{16} \\
\hline & $2000-2010$ & 5 & \\
\hline & Desde 2011 & 10 & \\
\hline Total & & & 5197 \\
\hline
\end{tabular}

\section{Discusión y Conclusiones}

El número de investigaciones que relacionan el autoconcepto y la inteligencia emocional con la actividad física deportiva va en aumento como se desprende de los resultados anteriores. Los estudios en lengua inglesa son muy abundantes en relación a los de lengua castellana, esta situación viene determinada porque la mayoría de los científicos de la actividad física y el deporte estudian los términos planteados en literatura anglosajona y también porque el número de revistas es mayor en este idioma.

En cuanto al autoconcepto y el deporte aparece un nú- mero escueto de trabajos si lo contrastamos con los de lengua inglesa, concretamente aparecen dos estudios (Fernández et al., 2010; Vaquero, Alacid, Muyor\& López-Miñarro, 2013), donde se realizan respectivamente una revisión bibliográfica en el primero y en el segundo se establecen las diferencias que existían entre el autoconcepto y las diversas modalidades de actividad física, empleándose para ello dos tipos de cuestionario, y que se aplicó a una muestra de 894 adolescentes, y se obtuvieron como principales resultados que las mujeres se preocupaban más por su apariencia física que por los hombres.

Al relacionar autoconcepto y actividad física, se encontraron siete trabajos, donde destacamos el de Álvarez, Cuevas, Lara \& González (2015), donde participaron 208 estudiantes se trató de identificar las diferencias entre practicantes y no de actividad física, denotándose la importancia que tenía la realización de práctica deportiva para los estudiantes.

En referencia a la Inteligencia Emocional y Deporte, se detectaron tres trabajos, donde destaca el presentado por García-Coll, Graupera, Ruiz-Pérez \&Palomo (2013), donde se validó un cuestionario que trataba sobre inteligencia emocional y deporte, le fue administrado a más de 2000 deportistas y se determinó que era un test fiable y que esta escala permite tener un inventario apto para medir la inteligencia emocional en el deporte.

En cuanto a la inteligencia emocional y actividad física se detectó un único estudio (Abad, Castillo \& Orizia, 2014), donde se analizaron a 35 mujeres que realizaban biodanza a los cuales se les administró el TIMMS-24, determinándose como la inteligencia emocional percibida aumentaba en el grupo de mujeres que desarrollaban esta actividad.

Al estudiar los vocablos en inglés, el número de estudios aumentó de manera considerable, de esta manera "Self-Concept Sport" obtuvo un total de 1861 entradas, siendo un tema muy tratado en los últimos años, de esta manera Ronkainen \& Ryba (2016), estudian la masculinidad dentro del hockey hielo, y de la percepción que se tiene sobre uno mismo, y las repercusiones de las lesiones en la historia de vida de algunos jugadores; del mismo modo Aasa, Paulin \& Madison (2017), analizan sobre 90 mujeres la importancia del autoconcepto sobre mujeres con un bajo nivel de condición física y sedentarias, y de cómo la realización de actividad física deportiva ocasiona un cambio en la aptitud después de participar en diversas tareas deportivas.

Al relacionar el "Self-Concept PhysicalActivity", se hallaron numerosos trabajos de investigación, entre ellos destacamos los de Muros, Cofre, Arriscado, Zurita \& Knox (2017), que analizaron en una población escolar chilena de 515 alumnos, los niveles de alimentación, condición física y autoconcepto, encontrándose relación entre todas las dimensiones, o el trabajo de Jekauc, Wagner, Hermann, Hegazy \& Woll (2017), que examina la relación existente entre habilida- 
des motoras y actividad física, para ello utilizaron un estudio de tipo longitudinal sobre 699 participantes, mostrando una concordancia indirecta entre ambas cuando se refería a las cualidades de fuerza, coordinación y flexibilidad. Estos autores concluyeron en que el autoconcepto de tipo físico es un elemento indispensable para la realización de actividad física de los adolescentes.

En el tema de la inteligencia emocional al aplicar los parámetros anglosajones "Emotional Intelligence Sport", el número de estudios se incrementa con respecto a los estudios en castellano, siendo temas abordados en los últimos años, en este sentido el trabajo de Laborde et al.(2017), estructura su estudio en el conocimiento de relación que puede existir entre la inteligencia emocional y su asociación con el deporte, para ello se analizaron 972 deportistas a los cuales se les aplicó una serie de instrumentos, no se apreciaron diferencias entre modalidades deportivas individuales o de equipo; en otro estudio Cowden (2016), examinó las relaciones entre la inteligencia emocional y el afrontamiento en 151 atletas varones adolescentes, determinando que aquellos deportistas con mayor nivel de inteligencia emocional presentan un incremento en la capacidad de afrontamiento.

En el análisis de la "Emotional Intelligence Physical Activity" se constata un incremento de estudios con respecto al castellano, destacamos por ejemplo el de Abdolrezapour (2017), que indica la importancia de la inteligencia emocional dentro de diversos contextos, y que alude al nivel social, físico o académico. Para ello trabaja en una muestra de 63 estudiantes apreciando su estudio en diversas disciplinas, e indica una alta relación entre inteligencia emocional y nivel oral de los alumnos.

Por último se desarrolla la relación que pueda existir entre todos los parámetros objeto de estudio "Sport, Emotional Intelligence Physical Activity”, en ella se analizan 16 estudios, aumentando el número de ellos en relación con el castellano, por ejemplo Littrel \& Lee (2016),hablan del interés en el estilo de entrenamiento y de cómo los jóvenes atletas pueden estar influenciados por el mismo; asimismo hablan de la conexión entre inteligencia emocional y liderazgo, y para ello hablan de una revisión de la literatura; y el trabajo de LópezCazorla, Hernández-Mendo, Reigal \& Morales (2015), que analizaron el autoconcepto y su relación con las habilidades psicológicas en triatletas, denotándose en este estudio sobre 88 de ellos la importancia de este parámetro y la implicación en variables psicológicas.

Como conclusión se puede indicar que existe un mayor número de artículos en lengua inglesa que en castellano, asimismo los estudios relacionados con autoconcepto son más numerosos que los de inteligencia emocional, y cuando se buscan trabajos que contengan la totalidad de los parámetros antes citados, en castellano no se detectan trabajos, mientras que en inglés aparecen dieciséis. Este trabajo pone de manifiesto la necesidad de seguir trabajando en esta línea que permitirá ahondar más en el conocimiento de factores psicosociales en los deportistas.

\section{Referencias}

1. Aasa, U., Paulin, J., \& Madison, G. (2017).Correspondence between physical self-concept and participation in, and fitness change after, biweekly body conditioning classes in sedentary women. Journal of Strength and Conditioning Research, 31(2), 451-461.

2. Abad, M. T., Castillo, E., \& Orizia, A. C. (2014).Los efectos de un programa motor basado en la biodanza en relación con parámetros de inteligencia emocional en mujeres. Cuadernos de Psicología del Deporte, 14(1), 13-21.

3. Abdolrezapour, P. (2017). Improving learner's oral fluency computermediated emotional intelligence activities, RECALL, 29(1), 80-98.

4. Álvarez, L., Cuevas, R., Lara, A., \& González, J. (2015). Diferencias del autoconcepto físico en practicantes y no practicantes de actividad física en estudiantes universitarios. Cuadernos de Psicología del Deporte, 15(2), 27-34.

5. Bar-On, R. (2006). The Bar-Onmodel of emotional-social intelligence (ESI). Psicothema, 18(1), 13-25.

6. Bretón, S., Zurita, F., \& Cepero, M. (2016). La resiliencia como factor determinante en el deportivo. Revisión Bibliográfica. E-Balonmano. Revista de Ciencias del Deporte, 12(2), 79-88.

7. Bretón, S., Zurita, F., \& Cepero, M. (2017). Análisis de los constructos de autoconcepto y resiliencia, en jugadoras de baloncesto de categoría cadete. Revista de Psicología del Deporte, 26(1), 127-132.

8. Cowden, R. (2016). Mental toughness, emotional intelligence and coping effectiveness: an analysis of construct interrelatedness among, high-performing adolescent male athletes. Perceptual and Motors Skills,
123(3), 737-753.

9. De Tejada, M. (2010). Evaluación de la autoestima en un grupo de escolares de la Gran Caracas. Liberabit, 16(1), 95-103.

10. Fernández, G., Contreras, O., García, M., \& González, S. (2010).Physical self-concept depending on the kind of physical activity practiced and motivation to it. Revista Latinoamericana de Psicología, 42(2), 251263.

11. Ferrer-Caja, E. \&Weiss, M. R. (2000). Predictors of intrinsic motivation among adolescent students in physical education. Research Quarterly for Exercise and Sport, 71(3), 267-279.

12. Fox, K. R. (1988). The self-esteem complex and youth fitness.Quest, 40, 230-246.

13. Fuentes, P. A. (2014). Estado de salud percibido, autoconcepto físico y metas personales en mujeres físicamente activas. Tesis Doctoral: Universidad de Extremadura.

14. García, F. \&Musitu, G. (2001). Autoconcepto Forma 5. AF5. Manual. Madrid: TEA.

15. García-Coll, V., Graupera, J. L., Ruiz-Pérez, L.M., \& Palomo, M. (2013). Inteligencia emocional en el deporte: validación española del Schutte Self Report Inventory (SSRI) en deportistas españoles. Cuadernos de Psicología del Deporte, 13(1), 25-36.

16. Goleman, D. (1995). Emotional intelligence. New York: Kairós.

17. Gońi, E. (2009). El autoconcepto personal: estructura interna, medida y variabilidad. País Vasco: Servicio Editorial de la Universidad del País Vasco. 
18. Jekauc, D., Wagner, M., Hermann, C., Hegazy, K., \&Woll, A. (2017). Does physical self-conceptmediate the relationship between motor abilities and physical activity in adolescents and young adults. PLOS ONE, 12(1), e0168539.

19. Laborde, S., Guilien, F., \& Watson, M. (2017).Trait emotional intelligence questionnaire full form and short-form versions: links with sport participation frecuency and duration and type of sport practiced. Personality and Individual Differences, 108, 5-9.

20. Littrel, H. \& Lee, Y, H. (2016).Emotional intelligence and autonomysupportive interpersonal style in coaching: review of literature. Asian Journal of Physical Education and Sport Science, 4(2), 1-17.

21. López-Cazorla, R., Hernández-Mendo, A., Reigal, R. E., \& Morales, V. (2015).Relaciones entre el autoconcepto y el perfil psicológico deportivo en triatletas. Cuadernos de Psicología del Deporte, 15(2), 95-102.

22. Mayer, J.D., \&Salovey, P. (1997).What is emotional intelligence?.en P. Salovey y D. Sluyter (Eds.). Emotional development and emotional intelligence: educational implications (pp. 3-31). New York: Basic Books.

23. Muros, J. J., Cofre, C., Arriscado, D., Zurita, F., \& Knox, E. (2017). Mediterranean diet adherence is associated with lifestyle, physical fitness and mental wellness among 10 years old in Chile. Nutrition, 35, 87-92.

24. Pineda, C. (2012). Inteligencia emocional y bienestar personal en estudiantes universitarios de Ciencias de la Salud. Tesis Doctoral: Universidad de Málaga.

25. Reigal, R. E., Becerra, C. A., Hernández, A.,\& Martín, I. (2014). Relación del autoconcepto con la condición física y la composición corporal en una muestra de adolescentes. Anales de Psicología, 30(3), 1079-1085.

26. Revuelta, L. \& Esnaola, I. (2011). Clima familiar deportivo y autoconcepto físico en la adolescencia. European Journal of Education and Psychology, 4(1), 19-31.
27. Ronkainen, N., \&Ryba, T. (2016).Is hockey just a game? Contesting meaning of the ice hockey life projects through a career-threatening injury. Journal of Sports Sciences, 35(10), 923-928.

28. Ros-Martínez, A., Moya-Faz, F. J., \& Garcés de los Fayos, E. J. (2013) Inteligencia emocional y deporte: situación actual del estado de la investigación. Cuadernos de Psicología del Deporte, 13(1), 105-112.

29. Sánchez-Alcaraz, B. J., \& Mármol, A. G. (2014). Autoconcepto físico en una muestra de estudiantes de primaria y su relación con el género y la práctica deportiva extraescolar. E-balonmano.com: Revista de Ciencias del Deporte, $10(2)$

30. Shavelson, J., Hubner, J.J., \& Stanton, G.C. (1976).Self-concept: validation of construct interpretations. Review of Educational Research, 46(3), 407-442.

31. Soriano, J. A., Navas, L., \&Holgado, F. P. (2014).El autoconcepto físico y su relación con el género y la edad en estudiantes de educación física. Apunts. Educación Física y Deportes, 106(4), 36-41.

32. Strein, W. (1996).Advances in research on academic self-concept: Implications for school psychology. School Psychology Review, 22(2), $273-$ 285.

33. Vaquero, R., Alacid, F., Muyor, J. M., \&López-Miñarro, P. A. (2013). Imagen Corporal; Revisión Bibliográfica. Nutrición Hospitalaria, 28(1), 27-35.

34. Zeidner, M., Roberts, R. D., \& Matthews, G. (2008). The science of emotional intelligence: current consensus and controversies. European Psychologist, 13(1), 64-78.

35. Zurita, F., Castro, M., Álvaro, J. I., Rodríguez, S., \& Pérez, A. (2016). Autoconcepto, actividad física y familia: análisis de un modelo de ecuaciones estructurales. Revista de Psicología del Deporte, 25(1), $97-$ 104. 